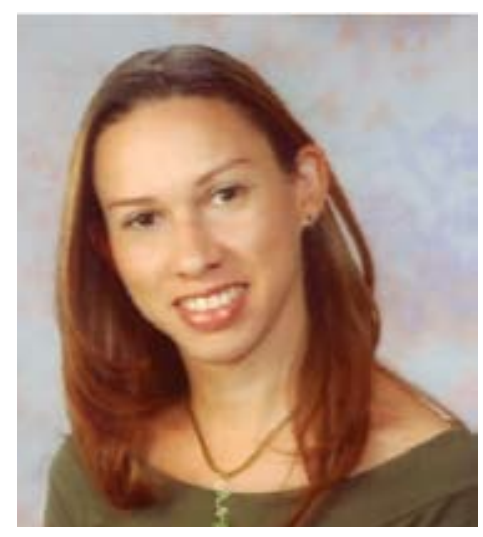

ECOS DESDELAS FACULTADES

\title{
LA COMPETENCIA DEL PROFESORADO DE LA PONTIFICIA UNIVERSIDAD CATÓLICA MADRE Y MAESTRA COMO FACTOR DE CALIDAD: UN ESTUDIO EMPÍRICO
}

\author{
Ginia Montes de Oca *
}

Este artículo está basado en la tesis doctoral “La Competencia Docente como factor de calidad de las universidades", realizada en la Universidad Complutense de Madrid. Se expone la experiencia de evaluación de las competencias de los docentes de la Pontificia Universidad Madre y Maestra por parte de los estudiantes. En éste se revisa, además, el concepto de competencias, el sentido y utilidad de la evaluación por competencias en las universidades, en especial la valoración realizada por los estudiantes.

Finalmente, se presentan los resultados de estudios diferenciales realizados en función de las facultades de esta Universidad.

\begin{abstract}
Introducción
Las Instituciones de Educación Superior desempeñan un papel de suma importancia en la formación de profesionales del más alto nivel y en la creación, avance, mejora, transferencia y adaptación de tecnologías, de modo que, lo que ellas hacen para responder de forma adecuada a los requerimientos de la sociedad actual, se constituye en un imperativo estratégico para el progreso nacional.
\end{abstract}

Para que la universidad pueda llevar a cabo una educación de calidad se debe tomar en cuenta el valor del profesorado, fundamental en esta misión, pues su formación académica y trayectoria profesional son aspectos que influyen en la calidad de la educación superior. El profesor orientador del aprendizaje es un guía que conduce al estudiante por el camino del saber sin imposiciones pero con la autoridad suficiente que emana de su experiencia y, sobre todo, con la confianza que en él han depositado sus alumnos, a partir del establecimiento de relaciones afectivas basadas en la aceptación, el respeto mutuo y la comprensión.

En este artículo se habla sobre la evaluación de las competencias del docente a partir de un estudio realizado en 4 universidades dominicanas',aunque sólo se presentan aquí los resultados obtenidos por la Pontificia
Universidad Católica Madre y Maestra (PUCMM). Este artículo está basado en la tesis doctoral "La Competencia Docente como factor de calidad de las universidades", realizada entre 2007 y 2009 en la Universidad Complutense de Madrid, Facultad de Educación, bajo la dirección de María José Fernández Díaz. En la indagación sobre el contexto se constató que las universidades dominicanas carecen de un sistema de evaluación docente consolidado, a la vez que no parecen manejar datos sobre las competencias docentes que inciden en la calidad de su docencia. Por tanto, los resultados obtenidos podrían proporcionar información relevante a la hora de diseñar proyectos de planificación y futuras intervenciones en la mejora docente.

Uno de los criterios que está siendo utilizado con mayor frecuencia para evaluar al profesorado universitario es el de competencia, que se entiende como los conocimientos, las actitudes, las habilidades, las aptitudes y las destrezas necesarias que posee un docente universitario para ejercer su labor. Las competencias tienen dos propósitos: 1) dan la posibilidad de diagnosticar la calidad de la actividad, la eficiencia de la labor docente y 2) certifican el nivel académico, profesional, tecnológico y científico del docente. Es competente el docente instruido, cuyos conocimientos han

\footnotetext{
* Licenciada en Psicología Escolar por la Universidad Autónoma de Santo Domingo. Magíster en Intervención Psicológica en Contextos Educativos por la Universidad Complutense de Madrid. Becada por la Fundación Carolina para estudios de doctorado sobre "Calidad y Evaluación de Instituciones, programas e Intervención Psicopedagógica" en esta última universidad.

1 Las universidades incluidas en el estudio fueron: Pontificia Universidad Madre y Maestra, Universidad Autónoma de Santo Domingo, Universidad Iberoamericana, y Universidad Organización y Métodos.
} 
sido comprobados y ha sido capaz de demostrar la experiencia adquirida en su quehacer. Según Fletcher (1997), el propósito de la evaluación es recoger suficientes evidencias de que los individuos pueden desempeñarse según las normas específicas en una función específica. Numerosos autores (Sanders \& Rivers, 1996; Wright, Horn \& Sanders, 1997; Hanushek, Kain \& Rivkin, 1998; Darling-Hammond, 1997, 2000; Klinger, 2000; Ellett \& Teddlie, 2003; Hattie, 2003; Wayne \& Youngs, 2003; McCaffrey et al, 2004; Hanushek \& Rivkin, 2006; Muñoz \& Chang, 2007) evidencian la importancia de evaluar la competencia del docente puesto que el desempeño de éstos afecta el aprendizaje de los alumnos. Ellos señalan que, para mejorar la educación, un factor decisivo lo constituye la intervención en la efectividad de los profesores.

Kleinhenz e Ingvarson (2004) mencionan que la fuerte relación entre la calidad de los profesores y el rendimiento de los alumnos demanda la existencia de sistemas efectivos que permitan la rendición de cuentas por parte de los profesores. La evaluación del desempeño docente permite entregar estímulos y reconocimientos a los docentes que destaquen por su desempeño (Wise et al, 1985; Mizala \& Romaguera, 2000b; Mizala \& Romaguera, 2002a; Mizala \& Romaguera 2003; Apablaza \& Bravo, 2007).

En las investigaciones realizadas con respecto a la valoración de los alumnos sobre la competencia docente se plantea que los estudiantes son las fuentes más cualificadas para informar sobre la medida en que su experiencia de aprendizaje fue productiva, informativa y si cumplió con sus expectativas. Es un hecho que un elevado porcentaje de docentes presentan una actitud moderadamente positiva sobre la validez y utilidad de la evaluación del profesorado por parte del alumnado como método para mejorar su docencia (Berk, 2005).

Investigadores como Aleamori (1978b), Cohen (1990), McKeachie (1979), Schmelkin \& Spencer (1997), Schum \& Vindra (1996), Steven \& Aleamori (1985), encontraron en sus estudios que la instrucción mejoraba significativamente cuando se les daba a los profesores un informe escrito sobre las valoraciones de sus estudiantes, ya que aquéllos suelen utilizar esta información para mejorar y enriquecer sus cursos. Por otra parte, para Tejedor (1990), la evaluación del profesor por sus estudiantes debería orientarse, fundamentalmente, a la estimación del nivel de calidad de la enseñanza universitaria, a fin de contribuir progresivamente a su mejora.

\section{Tipo de estudio}

Se tomó como punto de partida la clasificación en función de criterios que proponen Latorre, Del Rincón \& Arnal (1996). El estudio aquí presentado es de tipo exploratorio, ya que nos familiarizamos con la Competencia Docente del profesorado universitario de la República Dominicana, el cual ha sido un tópico poco analizado. A la vez, es descriptivo, pues tiene como objetivo el empleo de métodos descriptivos en el momento en que tienen lugar dichos acontecimientos.

De igual manera, podemos considerar el estudio cuantitativo porque se centra en aspectos observables que pueden ser cuantificados. También es de campo, porque se realiza en un ambiente natural, como es el de las aulas de clases. Por último, el estudio puede clasificarse como correlacional, ya que se

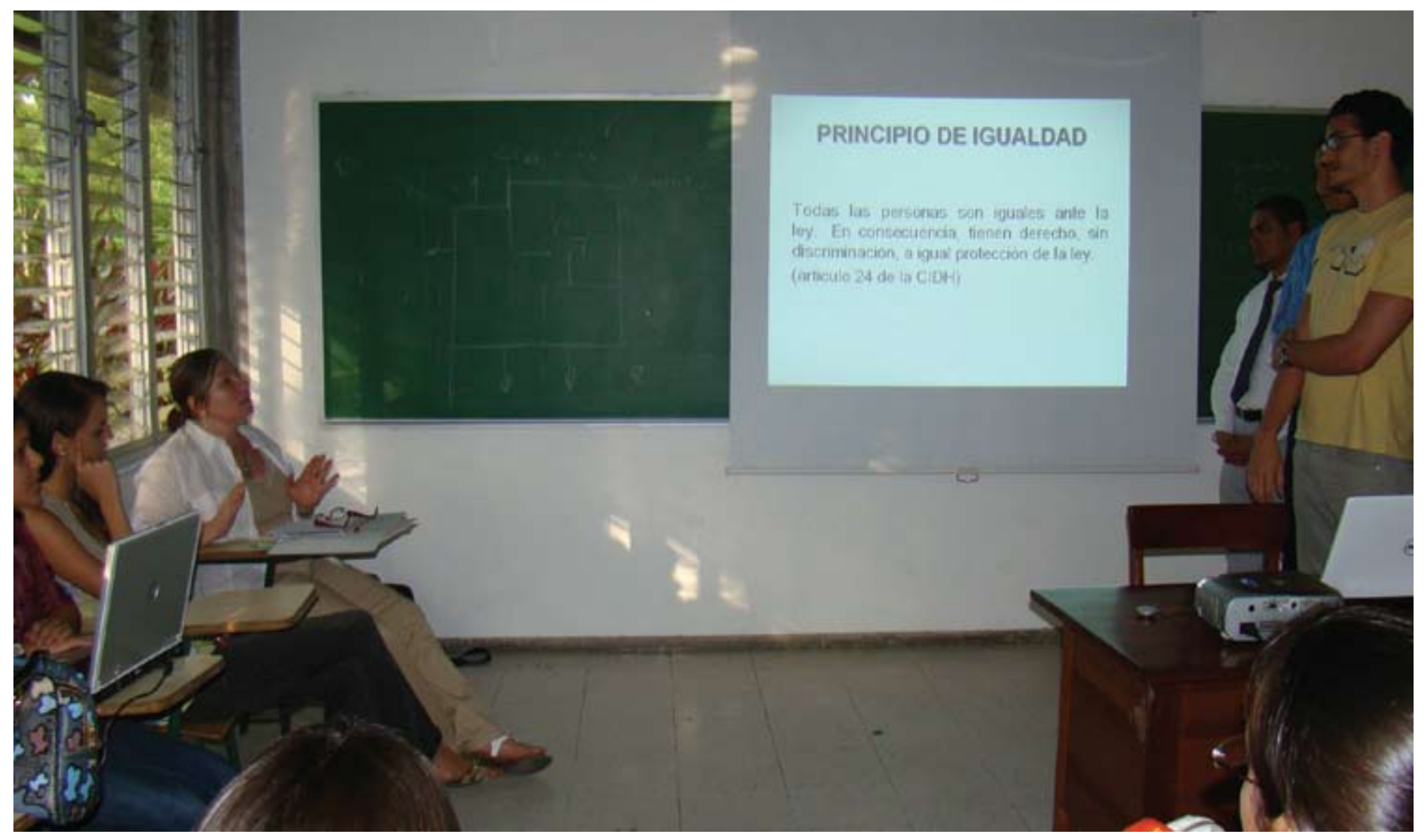


persigue determinar el grado en que las variaciones en uno o varios factores son concomitantes con la variación en otros; y es ex - post facto, porque este estudio no pretende establecer las razones de las creencias del alumno sobre las competencias de sus docentes sino, más bien, identificarlas y describirlas sistemáticamente, de manera que se constituyan en información útil para la comunidad académica.

\section{Objetivo}

El objetivo principal de esta investigación es analizar las competencias del docente de las universidades seleccionadas y los factores relacionados con dichas competencias, a través de un instrumento elaborado con rigor y sistematización que reuniera las características técnicas exigidas.

\section{Variables}

Una vez que se establecieron los objetivos de la investigación, se identificaron las variables que se consideraban relevantes para el estudio, las cuales están incluidas en el instrumento elaborado para medir las competencias docentes.

En las investigaciones sobre las competencias del docente universitario se plantea como una preocupación el hecho de seleccionar las variables implicadas en las mismas. Las variables seleccionadas están relacionadas con la actuación del docente en el aula. A continuación especificamos su estructura:

Planificación de la Docencia y Capacidad Pedagógica: es la forma en que el profesor diseña y planifica la docencia y el dominio de la asignatura que imparte. Incluye a) la integración del programa en el currículo, b) la organización de la asignatura y c) el dominio del tema.

Atención y Disposición con los Estudiantes: es la atención del docente con los estudiantes, así como el clima de clases y el respeto mutuo. Incluye a) la asesoría al alumno, b) la estimulación al alumno y c) las actitudes personales en las relaciones con los estudiantes.

Metodología Docente: es la forma en que el docente utiliza las estrategias de revisión, retroalimentación y análisis con sus estudiantes. Incluye a) las estrategias de retroalimentación y b) la interacción didáctica.

\section{Estrategias de Evaluación e Innovación didáctica y Tecnológica:} esta dimensión se refiere al uso de estrategias de evaluación, así como de recursos de apoyo a la docencia y recursos tecnológicos en el aula de clases. Incluye a) las estrategias de evaluación y b) los recursos didácticos y tecnológicos.

La selección de estas dimensiones responde a dos criterios. Primero, se desprende de la revisión de la literatura realizada al inicio de este estudio que hay dimensiones que poseen un peso determinante cuando medimos la competencia del docente. Segundo, viene dado por la validación de contenido que realizaron los expertos de distintas universidades españolas y dominicanas.

Para realizar este estudio, se diseñó y se elaboró un instrumento de medida consistente, el cual reúne las características exigidas para evaluar las competencias del docente universitario, y a

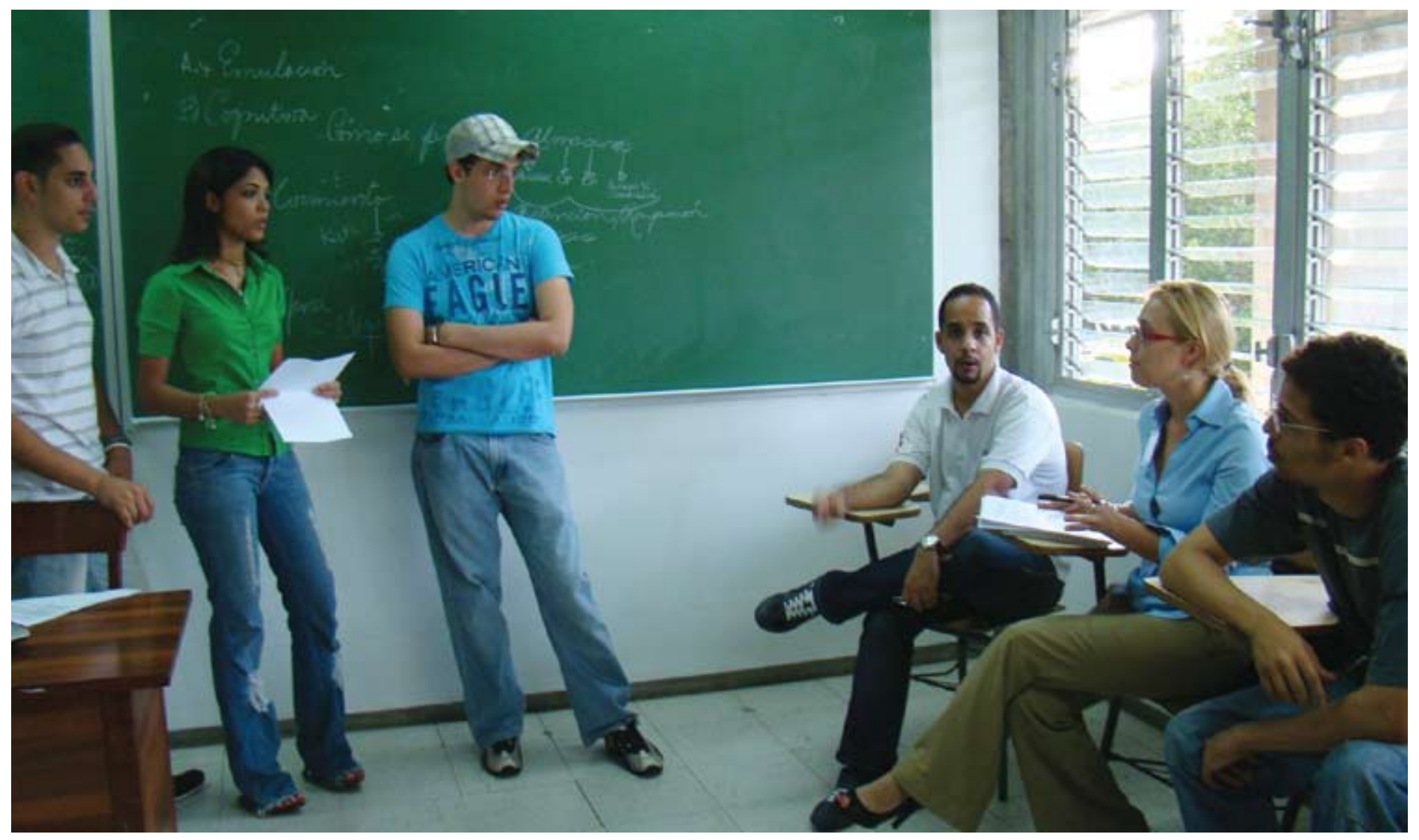


la vez, se identificaron las dimensiones subyacentes que la conforman. Se diseñó un instrumento al que se le dio el nombre de "Cuestionario para la evaluación de las competencias de los docentes universitarios", con 40 ítems de respuestas en escala Likert.

\section{Muestra}

La muestra seleccionada fue de 602 alumnos: 225 del Recinto Santo Tomás de Aquino y 377 del Campus de Santiago. Esta selección se realizó a través de un muestreo aleatorio incidental, que se caracteriza por un esfuerzo deliberado para obtener muestras "representativas" mediante la inclusión de grupos supuestamente típicos. Los porcentajes, junto a las facultades y sus respectivas carreras, aparecen en las tablas 1 y 2 :

Tabla 1. Facultades de la muestra

\begin{tabular}{lc}
\hline & Porcentaje válido \\
\hline Ciencias y Humanidades & 17,1 \\
Ciencias de la Salud & 23,6 \\
Ciencias Sociales & \\
y Administrativas & 42,5 \\
Ciencias de la Ingeniería & 16,8 \\
Total & 100,0
\end{tabular}

Tabla 2. Carreras de la muestra

\begin{tabular}{lc}
\hline Carrera & Porcentaje válido \\
\hline Administración & 11,6 \\
Arquitectura & 13,1 \\
Derecho & 4,8 \\
Educación & 4,0 \\
Ingeniería & 16,8 \\
Mercadeo & 11,0 \\
Medicina & 13,8 \\
Odontología & 9,8 \\
Psicología & 15,1
\end{tabular}

\section{Conclusiones asociadas al nivel de competencia y calidad de la docencia de los docentes de la PUCMM}

En relación a las puntuaciones globales obtenidas por esta Universidad, basadas en los factores evaluados que se relacionan con la competencia docente, presentamos la tabla 3, donde se muestran dichas puntuaciones y su significación en cuanto al nivel de desempeño. Podemos apreciar que las valoraciones son muy buenas, a excepción del factor Metodología Docente que obtiene una valoración media-aceptable.
Tabla 3. Puntuaciones globales por nivel de las dimensiones, en la PUCMM

\begin{tabular}{lcc}
\hline Dimensiones & Puntuación Global Nivel & \\
\hline $\begin{array}{l}\text { Planificación Docente } \\
\text { y Capacidades }\end{array}$ & & Alto \\
Pedagógicas & 4,18 & Alto \\
$\begin{array}{l}\text { Atención y Disposición } \\
\text { con los estudiantes }\end{array}$ & 4,07 & \\
$\begin{array}{l}\text { Estrategias de } \\
\text { Evaluación e Innovación } \\
\text { Didáctica y Tecnológica }\end{array}$ & 4,11 & Alto \\
Metodología Docente & 3,70 & Medio
\end{tabular}

Al realizar los estudios diferenciales por facultades por ANOVA², vemos con más detalles las puntuaciones obtenidas y el nivel de competencia de sus docentes.

EnlaPUCMM,lafacultaddeCienciasyHumanidadeseslaquemás se destaca en el factor Planificación de la Docenciay Capacidades Pedagógicas, ya que presenta diferencias significativas con las facultades de las Ciencias de la Ingeniería, Ciencias de la Salud y Ciencias Sociales y Administrativas. Los docentes de la Facultad de Ciencias de la Ingeniería de la PUCMM son los que poseen el nivel de desempeñomás bajo en lorelacionado conlaPlanificación de la Docencia y a las Capacidades Pedagógicas, ubicándose en la categoría de aceptable. Estos docentes deberían participar en programas de mejora de la calidad de la docencia. Los resultados los presentamos en la tabla 4.

\section{Tabla 4. Factor Planificación de la Docencia y Capacidades Pedagógicas}

\begin{tabular}{lcl}
\hline Dimensiones & Puntuación Global Nivel & \\
\hline Ciencias y Humanidades & 4.42 & Alto \\
Ciencias de la Ingeniería & 3.92 & Medio \\
Ciencias de la Salud & 4.18 & Alto \\
Ciencias Soc. y Adm. & 4.17 & Alto
\end{tabular}

Los resultados obtenidos en esta competencia son similares a la competencia anterior. Nuevamente, los docentes de la Facultad de Ciencias y Humanidades de la Pontificia Universidad Madre y Maestra, con relación al factor Atención y Disposición hacia los estudiantes muestran un nivel alto de competencia, presentando diferencias significativas a su favor con relación a la Facultad de las Ciencias de la Ingeniería. Asimismo, los docentes de la

\footnotetext{
2 ANOVA: (análisis de varianza factorial), es una prueba estadística para analizar las diferencias de la variable dependiente en función del efecto de una variable independiente con más de dos grupos. Este análisis consiste en determinar matemáticamente que parte de la varianza es atribuible a la variación que existe dentro de cada uno de los grupos y qué parte es atribuible a la variación de un grupo a otro (Latorre, del Rincón y Arnal, 1996).
} 
Facultad de Ciencias de la Ingeniería son los que presentan un nivel aceptable o medio de competencia. En este factor también aparecen candidatos para programas de mejora a la calidad de la docencia. A continuación, pueden verse los resultados, en la tabla 5.

Tabla 5. Factor Atención y Disposición con el estudiante

\begin{tabular}{lcl}
\hline Dimensiones & Puntuación Global Nivel & \\
\hline Ciencias y Humanidades & 4.34 & Alto \\
Ciencias de la Ingeniería & 3.81 & Medio \\
Ciencias de la Salud & 4.07 & Alto \\
Ciencias Soc. y Adm. & 4.05 & Alto
\end{tabular}

ConrelaciónalaMetodologíaDocente, los docentes delaFacultad de Ciencias y Humanidades vuelven a mostrar un nivel alto de desempeño, como se muestra en la tabla 6 . Los docentes de las demás facultades presentan un nivel aceptable, considerándose que los mismos deben formar parte de los grupos seleccionados para programas de formación y mejora de su calidad docente. La Facultad de Ciencias de la Ingeniería de la PUCMM es la que resulta con el nivel más bajo de las facultades, pero entra en el rango medio aceptable. En este caso los docentes de Ingeniería también ameritan intervención de programas de capacitación en cuanto a esta variable.

Tabla 6. Metodología Docente

\begin{tabular}{lcl}
\hline Dimensiones & Puntuación Global Nivel & \\
\hline Ciencias y Humanidades & 4.09 & Alto \\
Ciencias de la lngeniería & 3.44 & Medio \\
Ciencias de la Salud & 3.64 & Medio \\
Ciencias Soc. y Adm. & 3.68 & Medio
\end{tabular}

La PUCMM tiene docentes con un alto nivel de desempeño en cuanto a las Estrategias de Evaluación e Innovación Didáctica y Tecnológica, a excepción de los docentes de la Facultad de Ciencias de la Ingeniería que poseen un nivel de desempeño aceptable, tal como muestra la tabla 7. Las facultades de Ciencias y Humanidades, Ciencias de la Salud y Ciencias

Tabla 7. Factor Estrategias de Evaluación e Innovación Didáctica y Tecnológica

\begin{tabular}{lcl}
\hline Dimensiones & Puntuación Global Nivel & \\
\hline Ciencias y Humanidades & 4.41 & Alto \\
Ciencias de la Ingeniería & 3.82 & Medio \\
Ciencias de la Salud & 4.12 & Alto \\
Ciencias Soc. y Adm. & 4.10 & Alto
\end{tabular}

Sociales y Administrativas presentan diferencias significativas a su favor con la Facultad de Ciencias de la Ingeniería, siendo los docentes de las tres primeras facultades los que más destacan. Los docentes de la Facultad de Ciencias de la Ingeniería de la PUCMM deben ser integrados en programas de formación docente referidos a Estrategias de Evaluación e Innovación Didáctica y Tecnológica.

\section{Recomendaciones}

Las siguientes recomendaciones van dirigidas a todas las universidades que incluimos en el estudio. Planteamos algunas ideas que se pueden convertir en sencillas y efectivas acciones, al interior de cada universidad, para realizar mejoras en la competencia docente de su profesorado.

1. Crear un proyecto docente en el marco de la excelencia para que los profesores construyan sus propias concepciones sobre una docencia de calidad, lo cual ayudará a que ellos se sientan parte de la Institución y más comprometidos con la mejora de la calidad.

2. Iniciar un proceso de revisión y actualización de los perfiles por competencias de los docentes universitarios y, en caso de que no existan, crearlos. Esta medida es necesaria para implementar dispositivos de formación y entrenamiento que los comprometa a aumentar sus capacidades de observación, agudizar sus prácticas reflexivas, fortalecer su propia capacitación y fortalecer los valores.

3. Dimensionar el concepto de calidad de la docencia dentro de su contexto, teniendo en cuenta los distintos actores que la integran. A partir de esto, establecer criterios de calidad homogéneos dentro de las facultades y departamentos.

4. Estructurar un sistema de evaluación que, además de valorar las competencias de los docentes, permita la creación de un proceso de evaluación continua, rigurosa y sistemática. con el objetivo de desarrollar una cultura de la evaluación en sí misma.

5. Darle prioridad a la formación orientada a los procesos de innovación y cambio institucional.

6. Diseñar un plan de formación del profesorado sobre la evaluación sumativa y formativa, donde los docentes reconozcan el valor e importancia de dichas evaluaciones como mejora de la calidad universitaria.

7. Optimizar el manejo tanto de los recursos didácticos como los tecnológicos, con el propósito de que los docentes tengan acceso a trabajar en el aula con dichos recursos y, de esta misma forma, puedan dar respuesta a las necesidades de sus alumnos. Esto se logrará ofreciendo formación y apoyo al profesorado en cuanto a las estrategias didácticas y la incorporación de recursos tecnológicos.

8. Proporcionar a los docentes orientación sobre la relevancia e importancia de incorporar interacciones didácticas en la práctica educativa y sus consecuencias positivas. 
9. Proporcionar a los docentes orientación acerca de la importancia de la retroalimentación a los alumnos antes y después de las evaluaciones, a través de las interacciones didácticas.

10. Incentivar al docente sobre el uso de las nuevas tecnologías en su quehacer educativo, impartiendo cursos de formación y seguimiento continuo para que así puedan posicionarse a la vanguardia de los avances tecnológicos.

11. Construir redes docentes, para el intercambio de material y experiencias para, así, crear nuevos entornos pedagógicos.

\section{Referencias bibliográficas}

Apablaza, M., Bravo, M. \& Mozo, R. (2007). Evaluación docente: los cambios que se requieren. Santiago de Chile: Centro de Políticas Públicas, Universidad del Desarrollo. Extraído el 15 de abril de 2009, de http://www.udd.cl/prontus_facultades/site/artic/20090115/asocfile/informe_9.pdf

Escudero, T.; De Miguel, M.; Mora, J. G. \& Rodríguez, S. (1996). La Evaluación del profesorado. Un tema a debate. Revista de Investigación Educativa. 14 (2), 73-94.

Fletcher, S. (1997). Nuevas formas de evaluación y certificación. En: Competencia Laboral. Antología de Lecturas. México: CONOCER.

Marsh, H.W. (1989). Evaluación de la enseñanza por los estudiantes. En: T. Husen \& T.N. Postlenthwaite (Eds.) Enciclopedia Internacional de la Educación. Barcelona: Vicens Vives.

Marsh, H. W. (2007). Do university teachers become more effective with experience? A multilevel growth model of students' evaluations of teaching over 13 years. Journal of Educational Psychology, 99(4), 775-790.

Mizala, A. \& Romaguera, P. (2002). Equity and educational performance. Serie Economía No 136, Centro de Economía Aplicada, Departamento de Ingeniería Industrial, Facultad de Ciencias Físicas y Matemáticas, Universidad de Chile.

Yáñiz, C. (2008). Las competencias en el currículo universitario: implicaciones para diseñar el aprendizaje y para la formación del profesorado. Red U., Revista de Docencia Universitaria, número monográfico $1^{\circ}$. Extraído el 15 de abril de 2009 , dehttp://www.um.es/ead/Red_U/m1/yaniz.pdf

Zabalza, M. (2007): Competencias Docentes del Profesorado Universitario. Calidad y desarrollo profesional. Madrid. Narcea. 